UAB-FT-376

November, 1995

hep-ph/9511292

\title{
SUPERSYMMETRIC QCD CORRECTIONS TO THE TOP QUARK DECAY OF A HEAVY CHARGED HIGGS BOSON
}

\author{
Ricardo A. JIMÉNEZ, Joan SOLÀ \\ Grup de Física Teòrica \\ and \\ Institut de Física d'Altes Energies \\ Universitat Autònoma de Barcelona \\ 08193 Bellaterra (Barcelona), Catalonia, Spain
}

\begin{abstract}
The supersymmetric QCD corrections to the hadronic width of a heavy charged Higgs boson, basically dominated by the top-quark decay mode $H^{+} \rightarrow t \bar{b}$, are evaluated at $\mathcal{O}\left(\alpha_{s}\right)$ within the MSSM and compared with the standard QCD corrections. The study of such quantum effects, which turn out to be rather large, is essential to understand the hypothetical supersymmetric nature of a heavy charged Higgs boson potentially produced in the near future at the Tevatron and/or at the LHC.
\end{abstract}


After the discovery of the top quark at the Tevatron [1], the "next-to-leading" task in order of importance in Particle Physics is the search for Higgs bosons. Indeed, whereas the finding of the top quark completes the spectrum of matter fields in the Standard Model (SM) and it was a necessary condition for the SM to be correct, it is not at all a sufficient condition. The decisive proof lies in the nature of the spontaneous symmetry breaking mechanism (SSB). Thus the very crucial question is still left pending: is the SSB caused by truly fundamental scalars or is it triggered by a dynamical mechanism involving a new species of fermions and/or a new strong interaction force?. This question is as old as the proposal for the first serious extensions and alternatives to the SM, namely, Supersymmetry (SUSY) and Technicolour. Here we will be concerned with the Minimal Supersymmetric Standard Model (MSSM) [2].

In the near and middle future, with the upgrade of the Tevatron, the advent of the LHC, and the possible construction of an $e^{+} e^{-}$supercollider, new results on top quark physics, and perhaps also on Higgs physics, will be obtained in interplay with Supersymmetry that may be extremely helpful to complement the precious information already collected at LEP from $Z$-boson physics. Here we wish to dwell on the phenomenology of supersymmetric Higgs boson decays into hadrons with an eye on these future developments. Two basic parameters are needed to deal with the (tree-level) MSSM Higgs sector, namely $\left(M_{A^{0}}, \tan \beta\right)[3]$. In the present study (see also the companion paper [4]), it will be useful to divide the supersymmetric Higgs parameter space into two mass segments: light CP-odd higgses $\left(M_{A^{0}}<M_{Z}\right)$ and heavy CP-odd higgses $\left(M_{A^{0}}>M_{Z}\right)$. However, since these segments are unequally sensitive to large and small values of $\tan \beta$, it will prove convenient to define the following two relevant regions of the $\left(M_{A^{0}}, \tan \beta\right)$-parameter space. One of them, call it Region I, is characterized by Higgs masses in the light segment together with large values of $\tan \beta$ of order $\sim m_{t} / m_{b}$. The other region (Region II) consists of Higgs masses in the heavy segment in combination with more moderate values of $\tan \beta$, say in the interval $2 \lesssim \tan \beta \lesssim 30$ 円.

In Refs. [6, 7], whose notation and definitions we shall adopt hereafter, we studied the impact of Region I on the top-quark decay process. Here we will focus on a related process, namely, the top quark decay mode of a charged supersymmetric Higgs, $H^{+} \rightarrow t \bar{b}$, which is allowed in Region II, provided that $M_{A^{0}} \gtrsim 160 \mathrm{GeV}$. We shall again be partially concerned with Regions I and II in the companion paper [4], where we round off our study by addressing the quark-antiquark decay modes of the three neutral Higgs states of the MSSM.

1 Incidentally, we note that Regions I and II correspond (approximately) to domains of the $\left(M_{A^{0}}, \tan \beta\right)$-plane formerly used [5] to alleviate some of the -still not completely banished- $Z$-boson anomalies, where in addition it was required the concurrence of a chargino and a stop both as light as permitted by phenomenological bounds, namely, of about $60-70 \mathrm{GeV}$. 
While a simple tree-level study of $H^{+} \rightarrow t \bar{b}$ is blind to the nature of the Higgs sector to which $H^{+}$belongs, it is clear that a careful study of the quantum effects on $H^{+} \rightarrow t \bar{b}$ could be the clue to unravel the potential supersymmetric nature of the charged Higgs; in particular, it should be useful to distinguish it from a charged Higgs belonging to a general two-Higgs doublet model. The charged Higgs boson can decay hadronically into several quark final states, and if it is sufficiently heavy it can also decay into top and bottom quarks. It is natural to tackle the radiative effects on $H^{+} \rightarrow t \bar{b}$ by first considering the QCD corrections. The conventional QCD corrections were first considered in Ref.[\&] and they are known to be large and negative in the limit of Higgs masses much bigger than the quark masses. In spite of the huge size of the standard QCD corrections for light quark final states, it is clear that these channels are severely suppressed by the small Yukawa couplings $g m_{q} / M_{W} \ll 1$ (for $q=u, d, c, s$ ) and/or by off-diagonal CKM matrix elements, so that their branching fractions are very tiny. Therefore, as soon as the $t \bar{b}$ threshold is open $\left(M_{H^{+}}>m_{t}+m_{b} \sim 180 \mathrm{GeV}\right)$ one is left with $H^{+} \rightarrow t \bar{b}$ as the only relevant hadronic decay of a heavy charged Higgs boson. The conventional QCD corrections to that decay [8, 9] cannot distinguish the nature of the underlying Higgs model, but their knowledge is indispensable to probe the existence of additional sources of strong quantum effects beyond the SM. Here we will concentrate on the SUSY-QCD quantum effects mediated by squarks and gluinos and shall compare them with the standard QCD corrections. A few remarks on the remaining MSSM corrections will be made later on.

The relevant SUSY-QCD one-loop decay diagrams constructed from gluinos and squarks (stop and sbottom species) are seen in Fig.1a. In Fig.1b we sketch one possible mechanism that could be used at the LHC to annually produce $\sim 10^{4}-10^{6}$ charged Higgs particles of a mass comprised between a few hundred $\mathrm{GeV}$ up to about $1 \mathrm{TeV}$ for a luminosity of $\mathcal{L} \sim 10^{34} \mathrm{~cm}^{-2} s^{-1}[10$. There are both the $t \bar{b}$ - and $b \bar{t}$-fusion mechanisms, which are to be treated together with the processes $g \bar{b} \rightarrow H^{+} \bar{t}$ and $g b \rightarrow H^{-} t$ as carefully explained in [11]. Trigging on a top quark in association is very useful to avoid the signal being swamped by huge backgrounds. These mechanisms are primarily initiated by two highly energetic gluons, and use as a production vertex the very same decay vertex that we wish to study. We point out, in passing, that these diagrams also contribute to the cross-section for single top-quark production, whose measurement is one of the main goals at the next Tevatron run (Run II). It is not our aim to give the complete list of diagrams, but it is clear that these production mechanisms could be rather efficient in the colliders, for the $H^{+} t \bar{b}$-vertex can be strongly enhanced and result in a very distinctive phenomenology as compared to the experimental expectations for the SM Higgs production, typically through (one-loop) $g$-fusion [10]. Most important, in the MSSM the $H^{+} t \bar{b}$-vertex can receive significant corrections (in some cases of order $50 \%$ ) which could play a decisive 
role to disentangle whether a charged Higgs hypothetically produced in a hadron collider is supersymmetric or not.

To evaluate the relevant corrections to $\Gamma \equiv \Gamma\left(H^{+} \rightarrow t \bar{b}\right)$ in the MSSM, we shall adopt the on-shell renormalization scheme [12] where the fine structure constant, $\alpha$, and the masses of the gauge bosons, fermions and scalars are the renormalized parameters: $\left(\alpha, M_{W}, M_{Z}, M_{H}, m_{f}, M_{S U S Y}, \ldots\right)$. The interaction Lagrangian describing the $H^{+} t \bar{b}$ vertex in the MSSM reads as follows:

$$
\mathcal{L}_{H t b}=\frac{g V_{t b}}{\sqrt{2} M_{W}} H^{+} \bar{t}\left[m_{t} \cot \beta P_{L}+m_{b} \tan \beta P_{R}\right] b+\text { h.c. },
$$

where $P_{L, R}=1 / 2\left(1 \mp \gamma_{5}\right)$ are the chiral projector operators, $\tan \beta$ is the ratio between the vacuum expectation values of the two Higgs doublets of the MSSM [2] and $V_{t b}$ is the corresponding CKM matrix element-henceforth we set $V_{t b}=1$.

The on-shell renormalized vertex $H^{+} t \bar{b}$ in Fig.1a is derived following a straightforward generalization of standard procedures in the SM [12] and can be parametrized in terms of two form factors $H_{L}, H_{R}$ and the corresponding mass and wave-function renormalization counterterms $\delta m_{f}$ and $\delta Z_{L, R}^{f}$ associated to the external quark lines in Fig.1a:

$$
i G=\frac{i g}{\sqrt{2} M_{W}}\left[m_{t} \cot \beta\left(1+G_{L}\right) P_{L}+m_{b} \tan \beta\left(1+G_{R}\right) P_{R}\right]
$$

with

$$
\begin{aligned}
G_{L} & =H_{L}+\frac{\delta m_{t}}{m_{t}}+\frac{1}{2} \delta Z_{L}^{b}+\frac{1}{2} \delta Z_{R}^{t}, \\
G_{R} & =H_{R}+\frac{\delta m_{b}}{m_{b}}+\frac{1}{2} \delta Z_{L}^{t}+\frac{1}{2} \delta Z_{R}^{b} .
\end{aligned}
$$

The counterterms are computed in the on-shell scheme from the SUSY-QCD interaction Lagrangian involving squarks and gluinos [2]. The explicit results, which we refrain from repeating here, are common to those in Ref. [7]. As for the vertex form factors, they depend not only on pure strong supersymmetric interactions but also on the Higgs-stopsbottom semiweak interaction Lagrangian [2]. One finds (summation is understood over indices $a, b=1,2)$ :

$$
\begin{aligned}
& H_{L}=8 \pi \alpha_{s} i C_{F} \frac{G_{a b}^{*}}{m_{t} \cot \beta}\left[R_{1 b}^{(t)} R_{1 a}^{(b) *}\left(C_{11}-C_{12}\right) m_{t}+R_{2 b}^{(t)} R_{2 a}^{(b) *} C_{12} m_{b}+R_{2 b}^{(t)} R_{1 a}^{(b *)} C_{0} m_{\tilde{g}}\right] \\
& H_{R}=8 \pi \alpha_{s} i C_{F} \frac{G_{a b}^{*}}{m_{b} \tan \beta}\left[R_{2 b}^{(t)} R_{2 a}^{(b) *}\left(C_{11}-C_{12}\right) m_{t}+R_{1 b}^{(t)} R_{1 a}^{(b) *} C_{12} m_{b}+R_{1 b}^{(t)} R_{2 a}^{(b) *} C_{0} m_{\tilde{g}}\right]
\end{aligned}
$$

where $C_{\ldots}=C_{\ldots}\left(p, p^{\prime}, m_{\tilde{g}}, m_{\tilde{t}_{b}}, m_{\tilde{b}_{a}}\right)$ are standard three-point functions [6] also carrying indices $a, b$ summed over; $C_{F}=\left(N_{C}^{2}-1\right) / 2 N_{C}=4 / 3$ is a colour factor obtained after summation over colour indices, and

$$
G_{a b}=R_{1 a}^{(b) *} R_{1 b}^{(t)} g_{L L}+R_{2 a}^{(b) *} R_{2 b}^{(t)} g_{R R}+R_{2 a}^{(b) *} R_{1 b}^{(t)} g_{L R}+R_{1 a}^{(b) *} R_{2 b}^{(t)} g_{R L},
$$


with

$$
\begin{aligned}
& g_{L L}=M_{W}^{2} \sin 2 \beta-\left(m_{t}^{2} \cot \beta+m_{b}^{2} \tan \beta\right), \\
& g_{R R}=-m_{t} m_{b}(\tan \beta+\cot \beta), \\
& g_{L R}=-m_{b}\left(\mu+A_{b} \tan \beta\right), \\
& g_{R L}=-m_{t}\left(\mu+A_{t} \cot \beta\right) .
\end{aligned}
$$

The $2 \times 2$ rotation matrices

$$
R^{(q)}=\left(\begin{array}{cc}
\cos \theta_{q} & -\sin \theta_{q} \\
\sin \theta_{q} & \cos \theta_{q}
\end{array}\right) \quad(q=t, b),
$$

plaguing the previous formulae relate the weak-eigenstate squarks $\tilde{q}_{a}^{\prime}=\left\{\tilde{q}_{L}, \tilde{q}_{R}\right\}$ to the mass-eigenstates $\tilde{q}_{a}=\left\{\tilde{q}_{1}, \tilde{q}_{2}\right\}$ as follows: $\tilde{q}_{a}^{\prime}=\sum_{b} R_{a b}^{(q)} \tilde{q}_{b}$. Therefore the $R^{(q)}$ diagonalize the corresponding stop and sbottom mass matrices, whose standard form is well-known [2] but we will quote here explicitly for convenience:

$$
\begin{gathered}
\mathcal{M}_{\tilde{t}}^{2}=\left(\begin{array}{cc}
M_{\tilde{t}_{L}}^{2}+m_{t}^{2}+\cos 2 \beta\left(\frac{1}{2}-\frac{2}{3} s_{W}^{2}\right) M_{Z}^{2} & m_{t} M_{L R}^{t} \\
m_{t} M_{L R}^{t} & M_{\tilde{t}_{R}}^{2}+m_{t}^{2}+\frac{2}{3} \cos 2 \beta s_{W}^{2} M_{Z}^{2} \cdot
\end{array}\right), \\
\mathcal{M}_{\tilde{b}}^{2}=\left(\begin{array}{cc}
M_{\tilde{b}_{L}}^{2}+m_{b}^{2}+\cos 2 \beta\left(-\frac{1}{2}+\frac{1}{3} s_{W}^{2}\right) M_{Z}^{2} & m_{b} M_{L R}^{b} \\
m_{b} M_{L R}^{b} & M_{\tilde{b}_{R}}^{2}+m_{b}^{2}-\frac{1}{3} \cos 2 \beta s_{W}^{2} M_{Z}^{2},
\end{array}\right),
\end{gathered}
$$

with

$$
M_{L R}^{t}=A_{t}-\mu \cot \beta, \quad M_{L R}^{b}=A_{b}-\mu \tan \beta .
$$

From the renormalized amplitude (2) the width of $H^{+} \rightarrow t \bar{b}$, including the one-loop SUSY-QCD corrections, is the following:

$$
\Gamma=\Gamma_{0}\left\{1+\frac{U_{L}}{D}\left[2 \operatorname{Re}\left(G_{L}\right)\right]+\frac{U_{R}}{D}\left[2 \operatorname{Re}\left(G_{R}\right)\right]+\frac{U_{L R}}{D}\left[2 \operatorname{Re}\left(G_{L}+G_{R}\right)\right]\right\},
$$

where the lowest-order result is

$$
\Gamma_{0}=\left(\frac{N_{C} G_{F}}{4 \pi \sqrt{2}}\right) \frac{D}{M_{H^{+}}} \lambda^{1 / 2}\left(1, \frac{m_{t}^{2}}{M_{H^{+}}^{2}}, \frac{m_{b}^{2}}{M_{H^{+}}^{2}}\right),
$$

with $\lambda\left(1, x^{2}, y^{2}\right)=\left[1-(x+y)^{2}\right]\left[1-(x-y)^{2}\right]$, and

$$
\begin{aligned}
D & =\left(M_{H^{+}}^{2}-m_{t}^{2}-m_{b}^{2}\right)\left(m_{t}^{2} \cot ^{2} \beta+m_{b}^{2} \tan ^{2} \beta\right)-4 m_{t}^{2} m_{b}^{2}, \\
U_{L} & =\left(M_{H^{+}}^{2}-m_{t}^{2}-m_{b}^{2}\right) m_{t}^{2} \cot ^{2} \beta \\
U_{R} & =\left(M_{H^{+}}^{2}-m_{t}^{2}-m_{b}^{2}\right) m_{b}^{2} \tan ^{2} \beta, \\
U_{L R} & =-2 m_{t}^{2} m_{b}^{2} .
\end{aligned}
$$

The numerical analysis of the strong supersymmetric corrections to $\Gamma\left(H^{+} \rightarrow t \bar{b}\right)$ is presented in Figs.2-5. In all figures where the Higgs mass is fixed, we take $M_{H^{+}}=250 \mathrm{GeV}$, and we define $\alpha_{s}=\alpha_{s}\left(M_{H^{+}}\right)$by means of the (one-loop) expression

$$
\alpha_{s}\left(M_{H^{+}}\right)=\frac{6 \pi}{\left(33-2 n_{f}\right) \log \left(M_{H^{+}} / \Lambda_{n_{f}}\right)},
$$


normalized as $\alpha_{s}\left(M_{Z}\right) \simeq 0.12$, where $n_{f}$ is the number of quark flavors with threshold below the Higgs boson mass $M_{H^{+}}$.

We treat the sbottom mass matrix (9) assuming a non-zero mixing parameter $M_{L R}^{b}$. Once $\mu$ and $\tan \beta$ are given, we use $A_{b}$ as one of the inputs. However, for the sake of simplicity we assume that $\theta_{b}=\pi / 4$, so that the two diagonal entries are equal to a common value. We denote by $m_{\tilde{b}_{1}}$ the lightest sbottom mass-eigenvalue and take it as the remaining input; typically, it is bound to satisfy $m_{\tilde{b}_{1}} \gtrsim 100-150 \mathrm{GeV}$ from collider data, but the limits are not so stringent as those from LEP, namely $m_{\tilde{b}_{1}} \gtrsim 65 \mathrm{GeV}$. As for the stop mass matrix, we choose our inputs as follows. In this case the lightest stop mass $m_{\tilde{t}_{1}}$ is strictly limited only by the LEP 1.5 bound $m_{\tilde{t}_{1}} \gtrsim 65 \mathrm{GeV}$, and we take it as one of the inputs. Thus, upon fixing $\mu, \tan \beta$ and taking into account that $S U(2)_{L}$-gauge invariance requires $M_{\tilde{t}_{L}}=M_{\tilde{b}_{L}}$, it follows that the stop mass matrix depends on one remaining parameter which can be taken as $A_{t}$.

To start with the numerical analysis, we study the dependence of the SUSY-QCD effects on the crucial parameter $\tan \beta$. In Fig.2a we plot the SUSY-QCD corrected width, eq.(11), versus $\tan \beta$, for fixed values of the other parameters. For completeness, we have included in this figure the partial widths of the alternative decays $H^{+} \rightarrow \tau^{+} \nu_{\tau}$ and $H^{+} \rightarrow W^{+} h^{0}$, which are obviously free of $\mathcal{O}\left(\alpha_{s}\right)$ QCD corrections. (To avoid cluttering, we have not included $H^{+} \rightarrow c \bar{s}$; it is overwhelmed by the $\tau$-lepton mode as soon as $\tan \beta \gtrsim 2$.) It is patent from Fig.2a that, for charged Higgs masses above the $t \bar{b}$ threshold, the decay $H^{+} \rightarrow t \bar{b}$ is dominant. Only for very large $\tan \beta(>30)$ and for sufficiently big and positive $\mu(\mu>100 \mathrm{GeV})$-hence outside Region II - the negative corrections to $H^{+} \rightarrow t \bar{b}$ are huge enough to drive its partial width down to the level of $H^{+} \rightarrow \tau^{+} \nu_{\tau}$. Therefore, within the limits of Region II, the top quark decay of the charged Higgs is, by far, the most relevant decay mode to look at.

In considering the various parameter dependences, we present the results of our analysis in terms of the quantity

$$
\delta_{\tilde{g}}=\frac{\Gamma-\Gamma_{0}}{\Gamma_{0}},
$$

which gives the relative correction with respect to the tree-level width. At large $\tan \beta$, the role played by the bottom quark mass becomes very important. Indeed, in Fig.2b we confirm that the external self-energies (basically the one from the $b$-line) give the bulk of the corrections displayed in Fig.2a, whereas the (finite) vertex effect is comparatively much smaller and its yield becomes rapidly saturated. The existence of potentially large SUSY-QCD corrections in the decay $H^{+} \rightarrow t \bar{b}$ could be foreseen from the work of Ref. 13, where it is shown that the SUSY-QCD bottom mass corrections are proportional to $\mu \tan \beta$ (for fixed, nonvanishing, $\mu$ and large $\tan \beta$ ). In our case, these corrections are fed into the counterterm $\delta m_{b} / m_{b}$ on eq.(3) and, when viewed in terms of diagrams of the electroweak- 
eigenstate basis, they appear as finite contributions proportional to $M_{L R}^{b}$ generated from squark-gluino loopst.

From Fig.3a we read off the incidence of the parameter $\mu$ on $\delta_{\tilde{g}}$ for various $\tan \beta$. We see that the SUSY-QCD correction is extremely sensitive to $\mu$ both on its value and on its sign. For this reason we have explored a moderate range of $\mu$ values. It turns out that the sign of the SUSY-QCD correction is basically opposite to the sign of $\mu$, and the respective corrections for $+\mu$ and for $-\mu$ take on approximately the same absolute value.

Worth noticing is also the dependence on the gluino mass (Fig.3b). The various steep falls in that figure are associated to the presence of threshold effects occurring at points satisfying $m_{\tilde{g}}+m_{\tilde{t}_{1}} \simeq m_{t}$. An analogous situation was observed in Ref. [6] for the SUSY corrections to the standard top-quark decay. Away from the threshold points, the behaviour of $\delta_{\tilde{g}}$ is smooth and perfectly consistent with perturbation theory. On the other hand, the sensitivity on $m_{\tilde{t}_{1}}$ is not dramatic, as can also be appraised in Fig.3b. This is to be expected from the fact mentioned above that it is the bottom (not the top) selfenergy that dominates the corrections. Virtually for any $m_{\tilde{t}_{1}}$, there emerges an important correction which raises a long way with the gluino mass before bending -very gently into the decoupling regime, as we have checked. The fact that the decoupling rate of the gluinos appears to be so slow has an obvious phenomenological interest.

In Fig.4a we analyze in detail the SUSY-QCD correction as a function of the sbottom masses. We find convenient to plot $\delta_{\tilde{g}}$ versus $m_{\tilde{b}_{1}}$, for fixed values of the other parameters and for various $\tan \beta$. We see that even for $m_{\tilde{b}_{1}}$ exceeding $250 \mathrm{GeV}$, and $\tan \beta>10$, the correction remains sizeable $\left(\delta_{\tilde{g}}>10 \%\right)$. As another feature, in Fig. $4 \mathrm{~b}$ we realize that $\delta_{\tilde{g}}$ is not symmetric with respect to the sign of $A_{b}$. Once the sign $\mu<0$ is chosen, the correction is larger for negative values of $A_{b}$ than for positive values. We have erred on the conservative side by choosing $A_{b}=+300 \mathrm{GeV}$ wherever this parameter is fixed.

In Fig.5 we compare the one-loop SUSY-QCD corrections with the standard $\mathcal{O}\left(\alpha_{s}\right)$ QCD corrections as a function of $M_{H^{+}}$and different values of $\tan \beta$. For the latter corrections we use the full analytical formulae of Ref. [9] In the limit $M_{H^{+}} \gg m_{t}$, the standard QCD correction boils down to the simple expression

$$
\delta_{g}=\frac{\Gamma_{Q C D}-\Gamma_{0}}{\Gamma_{0}}=\left(\frac{C_{F} \alpha_{s}}{2 \pi}\right) \frac{m_{t}^{2} \cot ^{2} \beta\left(\frac{9}{2}-6 \log \frac{M_{H^{+}}}{m_{t}}\right)+m_{b}^{2} \tan ^{2} \beta\left(\frac{9}{2}-6 \log \frac{M_{H^{+}}}{m_{b}}\right)}{m_{t}^{2} \cot ^{2} \beta+m_{b}^{2} \tan ^{2} \beta} .
$$

This formula is very convenient to understand the asymptotic behaviour. However, as we have checked, it is inaccurate for the present range of values of $m_{t}$ unless $M_{H^{+}}$is

\footnotetext{
${ }^{2}$ In the absence of sbottom mixing, i.e. $M_{L R}^{b}=0$, the large contribution $\delta m_{b} / m_{b} \sim-\mu \tan \beta$ is no longer possible, but then the vertex correction does precisely inherits this behaviour and compensates for it. However, the condition $M_{L R}^{b}=0$, combined with a large value of $\tan \beta$, leads to an scenario characterized by a value of $A_{b}$ which overshoots the natural range expected for this parameter 14.

${ }^{3}$ We have corrected several misprints on eq.(5.2) of Ref. [9].
} 
extremely large (beyond $1 \mathrm{TeV}$ ).

Remarkably, we recognize from Fig.5 that the supersymmetric QCD effects $\left(\delta_{\tilde{g}}\right)$ can be comparable or even larger than the conventional QCD corrections $\left(\delta_{g}\right)$. For a given $\tan \beta$, the relative size of the SUSY-QCD effects versus the standard QCD effects depends on the value of $M_{H^{+}}$. Notwithstanding, it is clear that $\delta_{\tilde{g}}$ remains fairly insensitive to $M_{H^{+}}$.

Some discussion on previous work is in order. A first study of the SUSY-QCD corrections to the hadronic width of a charged Higgs boson is performed in Refs. [15]. However, these references either use a too simplified set of assumptions on the spectrum of gluinos and squarks (e.g. all squarks are assumed unmixed and degenerate) and/or $m_{b}=0$ is assumed, so that the local behaviours of $\delta_{\tilde{g}}$ and the crucial effect from finite bottom mass corrections at large $\tan \beta$ are fully unnoticed.

Although we have concentrated on the computation of the QCD corrections, it is legitimate to worry about the larger and far more complex body of electroweak quantum effects, especially those coming from possible enhanced Yukawa couplings. A full analysis is under way and will be presented elsewhere [16], but a few comments may be necessary here. In fact, Yukawa couplings can also give contributions to $\delta m_{b} / m_{b}$ proportional to $\tan \beta$ [13], but their effect is in general smaller than in the SUSY-QCD case. For example, if there is no large hierarchy between the sparticle masses, the ratio between the SUSY-QCD and the Yukawa coupling contributions to $\delta m_{b} / m_{b}$ can be estimated (at high $\tan \beta)$ as $4 m_{\tilde{g}} / A_{t}$ times a slowly varying function of the masses of order 1 [13], where the (approximate) proportionality to the gluino mass reflects the aforementioned very slowly decoupling rate of the latter. In view of the present bounds on the gluino mass, and since $A_{t}$ (as well as $A_{b}$ ) cannot increase arbitrarily, we expect that the SUSY-QCD effects can be dominant, and even overwhelming for sufficiently heavy gluinos. Notwithstanding, the sole estimation in terms of $\delta m_{b} / m_{b}$ may be insufficient, for there are also plenty of additional electroweak vertex contributions both from the Higgs sector and from the stop-sbottom/chargino-neutralino sector where those Yukawa couplings are also involved. Hence, in contrast to the SUSY-QCD case, it is not obvious a priori what is the net outcome of the leading electroweak contributions. A partial evaluation of the Yukawa coupling effects from supersymmetric Higgs bosons has been made in Ref. 17] and entail only a few percent change in the partial width. Even though a complete calculation of the Higgs effects - not to mention those associated to the full plethora of supersymmetric particles - is not yet available in the literature, a preliminary analysis made by the authors [16] leads to the conclusion that the over-all leading electroweak supersymmetric effects, though not negligible, remain comparatively small and do not drastically alter the SUSY-QCD picture presented here.

In conclusion, we have presented a fairly complete treatment of the SUSY-QCD cor- 
rections to the partial width of the top quark decay of a charged Higgs boson and have put forward plenty of evidence that they can be rather large (typically between 10\% - 50\%), slowly decoupling and of both signs. Consequently, they can either reinforce the conventional QCD corrections or counterbalance them, and even reverse their sign; the QCD corrections would then be found much "larger", "missing" or with the "wrong" sign, respectively. This should be helpful to differentiate $H^{+}$from alternative charged pseudoscalar decays leading to the same final states. Ultimately, the precise knowledge of the quantum effects on $\mathrm{H}^{+} \rightarrow t \bar{b}$ should provide the characteristic features necessary to pin down the supersymmetric nature of a heavy charged Higgs hypothetically discovered in the next generation of experiments at the Tevatron and at the LHC. As we have shown, the mechanisms capable of producing a charged Higgs scalar in a hadron collider, e.g. $t \bar{b}$ and $b \bar{t}$ fusion, which can be greatly enhanced at large $\tan \beta$, are very sensitive to potentially large SUSY-QCD quantum effects. If these effects are eventually found, they could be the smoking gun needed to recognize that the produced $H^{ \pm}$in a hadron collider is, truly, a SUSY Higgs. After submiting this paper, we have noticed the work of Ref.[18] dealing with the same subject.

\section{Acknowledgements:}

One of us (J.S.) has benefited from conversations with M. Martínez and F. Palla. We are very grateful to J.A. Coarasa for a careful reading of the manuscript and for a numerical cross-check of the standard QCD contribution using an independent numerical code based on Mathematica. This work has been partially supported by CICYT under project No. AEN95-0882.

\section{References}

[1] F. Abe et al. (CDF Collab.), Phys. Rev. Lett. 74 (1995) 2626; S. Abachi et al. (D0 Collab.), Phys. Rev. Lett. 74 (1995) 2632.

[2] H. Nilles, Phys. Rep. 110 (1984) 1; H. Haber and G. Kane, Phys. Rep. 117 (1985) 75.

[3] J.F. Gunion, H.E. Haber, G.L. Kane, S. Dawson, The Higgs Hunters's Guide (Addison-Wesley, Menlo-Park, 1990).

[4] J. A. Coarasa, R.A. Jimenez, J. Solà, Strong effects on the hadronic widths of the neutral Higgs bosons of the MSSM, preprint UAB-FT-377, November 1995 hepph/9511402.

[5] D. Garcia, R.A. Jiménez, J. Solà, Phys. Lett. B 347 (1995) 321 [E: B 351 (1995) 602]; D. Garcia, J. Solà, Phys. Lett. B 354 (1995) 335; ibid B 357 (1995) 349. 
[6] D. Garcia, W. Hollik, R.A. Jiménez, J. Solà, Nucl. Phys. B 427 (1994) 53; A. Dabelstein, W. Hollik, R.A. Jiménez, C. Jünger, J. Solà, Nucl. Phys. B 456 (1995) 75 .

[7] J. Guasch, R.A. Jiménez, J. Solà, Phys. Lett. B 360 (1995) 47.

[8] C.S. Li, R. Oakes, Phys. Rev. D 43 (1991) 855; A. Méndez, A. Pomarol, Phys. Lett. B 252 (1990) 461.

[9] A. Djouadi, P. Gambino Phys. Rev. D 51 (1995) 218.

[10] S. Dawson, Z. Kunszt and J. F. Gunion, in: Perspectives on Higgs Physics, Advanced Series on Directions in High Energy Physics, Vol.13, ed. by G.L. Kane (World Scientific, Singapore, 1993).

[11] J.F. Gunion, H.E. Haber, F.E. Paige, W.K. Tung, S. Willenbrock, Nucl. Phys. B 294 (1987) 621; R.M. Barnett, H.E. Haber, D. Soper, Nucl. Phys. B 306 (1988) 697.

[12] M. Böhm, H. Spiesberger, W. Hollik, Fortschr. Phys. 34 (1986) 687; W. Hollik, Fortschr. Phys. 38 (1990) 165.

[13] L.J. Hall, R. Rattazzi, U. Sarid, Phys. Rev. D 50 (1994) 7048; M. Carena, S. Pokorski, C.E.M. Wagner, Nucl. Phys. B 426 (1994) 269.

[14] We thank our anonymous Referee for an insightful remark on this point.

[15] C.S. Li, J.M. Yang, Phys. Lett.B 315 (1993) 367; H. König, Phys. Rev. D 50 (1994) 3310 .

[16] J. A. Coarasa, D. Garcia, J. Guasch, R.A. Jiménez, J. Solà, preprint UAB-FT (in preparation).

[17] J.M. Yang, C.S. Li, B.Q. Hu, Phys. Rev. D 47 (1993) 2872.

[18] A. Bartl, H. Eberl, K. Hidaka, T. Kon, W. Majerotto, Y. Yamada, UWThPh1995-35 hep-ph/9511385.

\section{Figure Captions}

- Fig.1 (a) SUSY-QCD Feynman diagrams, up to one-loop order, correcting the partial width of $H^{+} \rightarrow t \bar{b}$; (b) Typical charged-Higgs production mechanism at hadron colliders. 
- Fig.2 (a) SUSY-QCD corrected $\Gamma\left(H^{+} \rightarrow t \bar{b}\right)$ - eq.(11) - (in $\left.G e V\right)$ as a function of $\tan \beta$, compared to the corresponding tree-level width, $\Gamma_{0}$. Also shown are the partial widths of the alternative decays $H^{+} \rightarrow \tau^{+} \nu_{\tau}$ and $H^{+} \rightarrow W^{+} h^{0}$. The topquark mass is $m_{t}=175 \mathrm{GeV}$; (b) Comparative effects from the (finite) vertex and self-energies as a function of $\tan \beta$. The fixed parameters for (a) and (b) are given in the frame.

- Fig.3 (a) Dependence of the relative SUSY-QCD correction $\delta_{\tilde{g}}$, eq.(15), upon the supersymmetric Higgs mixing mass parameter, $\mu$; (b) Evolution of the SUSY-QCD correction in terms of the gluino mass for $m_{\tilde{t}_{1}}=65,80,100 \mathrm{GeV}, \tan \beta=30$. Rest of inputs as in Fig.2.

- Fig.4 (a) $\delta_{\tilde{g}}$ as a function of $m_{\tilde{b}_{1}} ;$ (b) $\delta_{\tilde{g}}$ as a function of $A_{b}$. In both cases $\tan \beta=$ 10,20,30 and the remaining inputs are as in Fig.2

- Fig.5 The $\mathcal{O}\left(\alpha_{s}\right)$ standard QCD correction, $\delta_{g}$, compared to the SUSY-QCD correction, $\delta_{\tilde{g}}$, as a function of $M_{H^{+}}$and for two values of $\tan \beta$. Rest of inputs as in Fig.2. 

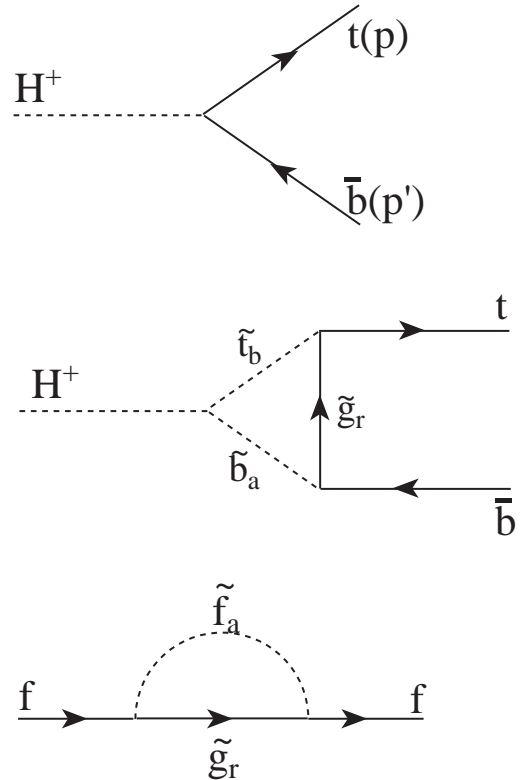

$(\mathrm{f}=\mathrm{b}, \mathrm{t})$

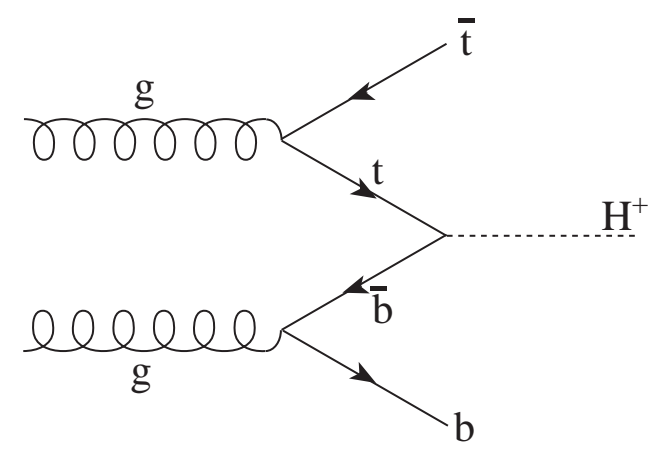

(a)

(b)

Fig. 1 

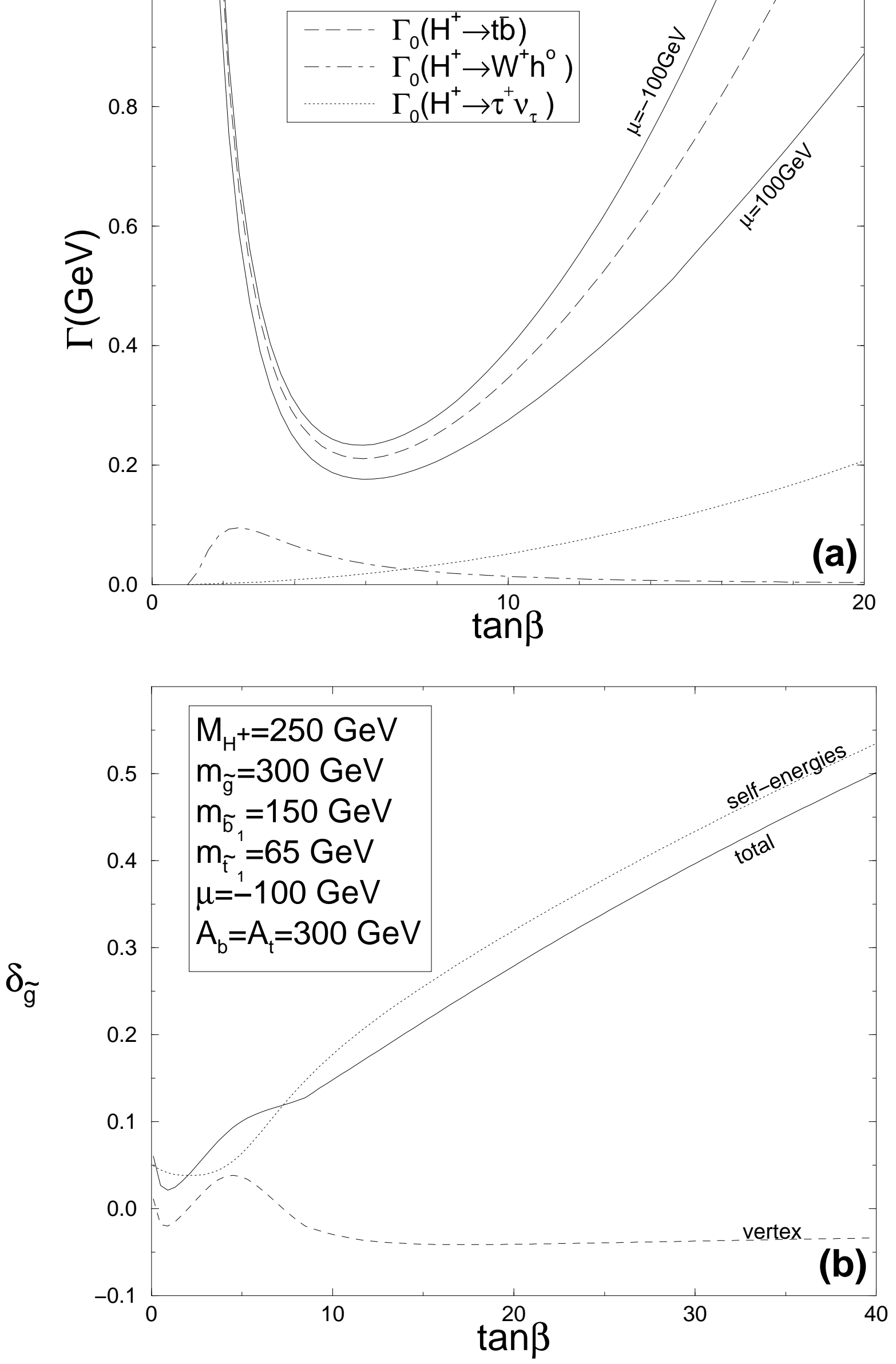

Fig. 2 

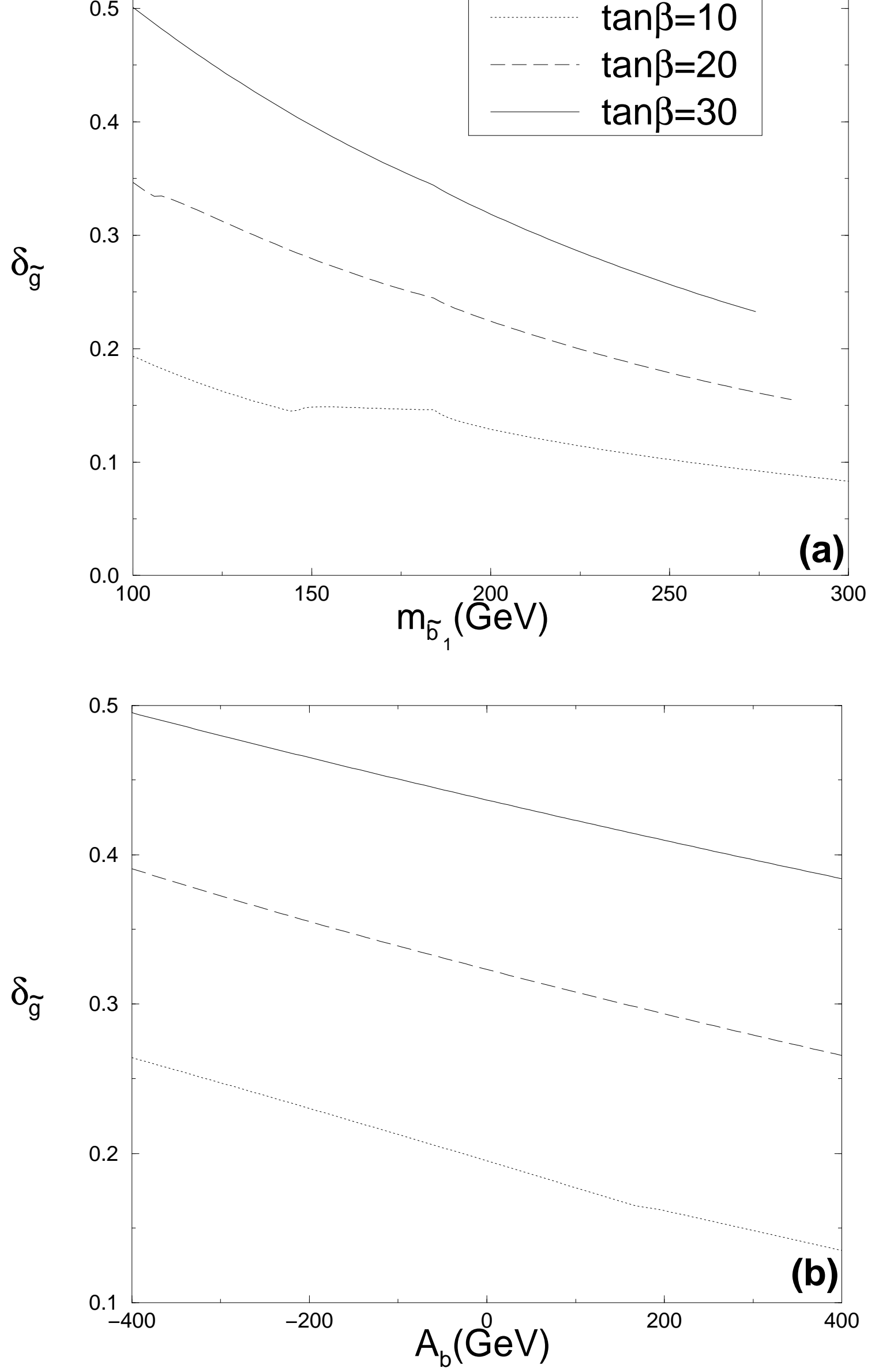

Fig. 4 


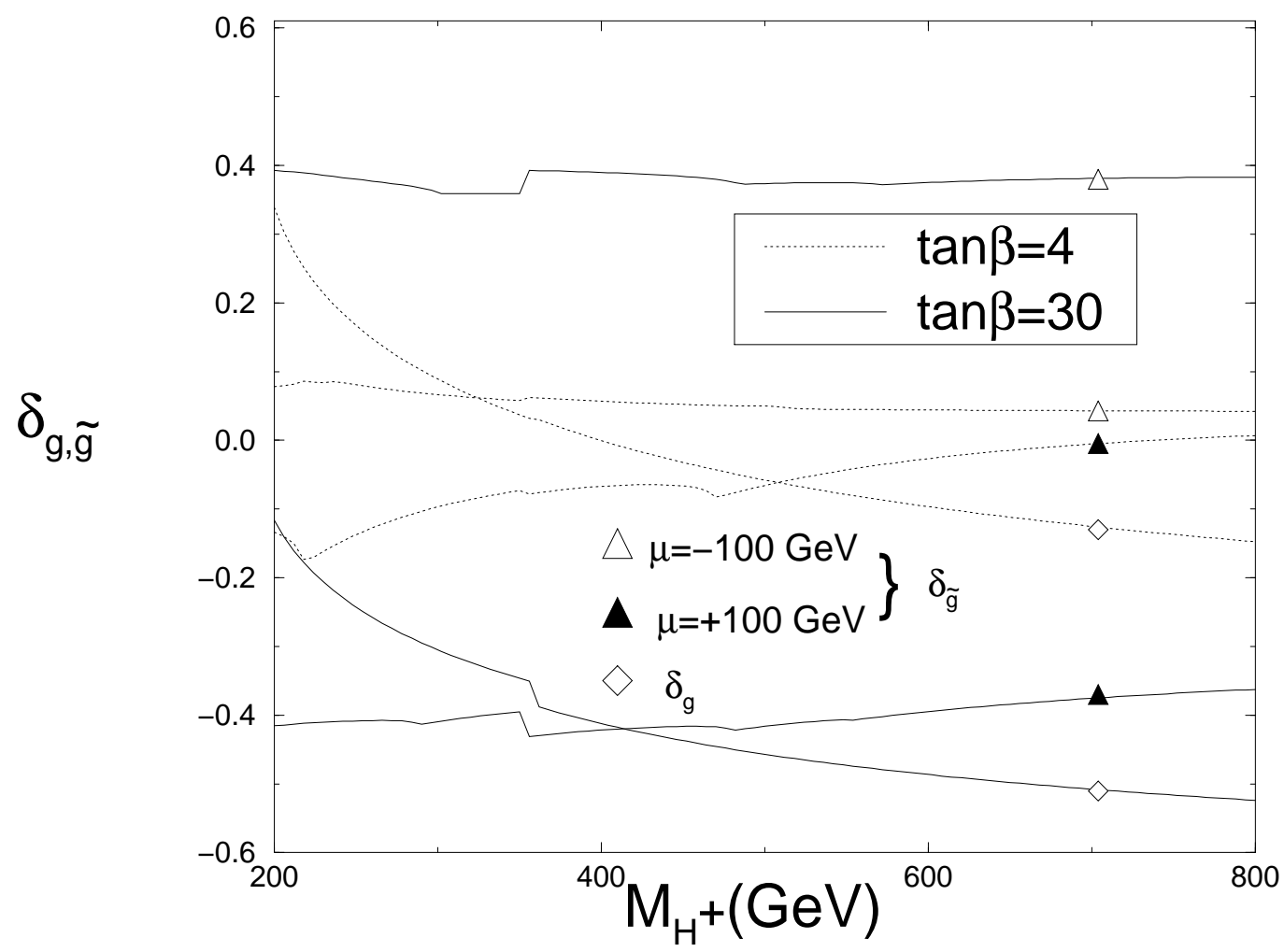

Fig. 5 